\title{
Flying lemurs - The 'flying tree shrews'? Molecular cytogenetic evidence for a Scandentia-Dermoptera sister clade Wenhui $\mathrm{Nie}^{1}$, Beiyuan Fu² ${ }^{2}$ Patricia CM O'Brien ${ }^{2}$, Jinhuan Wang1, Weiting $\mathrm{Su}^{1}{ }^{1}$, Alongkoad Tanomtong ${ }^{3}$, Vitaly Volobouev ${ }^{4}$, Malcolm A Ferguson-Smith ${ }^{2}$ and Fengtang Yang*5
}

Address: 'State Key Laboratory of Genetic Resources and Evolution, Kunming Institute of Zoology, Chinese Academy of Sciences, Kunming, Yunnan 650223, People's Republic of China, ${ }^{2}$ Cambridge Resource Centre for Comparative Genomics, Department of Veterinary Medicine, University of Cambridge, Madingley Road, Cambridge CB3 OES, UK, ${ }^{3}$ Department of Biology, Faculty of Science, Khon Kaen University, Khon Kaen 40002, Thailand, ${ }^{4}$ Muséum National d'Histoire Naturelle, Origine, Structure et Evolution de la Biodiversité, rue Buffon, 75005 Paris, France and ${ }^{5}$ Wellcome Trust Sanger Institute, Wellcome Trust Genome Campus, Hinxton, Cambridge CB10 1SA, UK

Email: Wenhui Nie - whnie@mail.kiz.ac.cn; Beiyuan Fu - bf204@cam.ac.uk; Patricia CM O'Brien - pco20@mole.bio.cam.ac.uk; Jinhuan Wang - goldjing2007@yahoo.cn; Weiting Su - su3.1010@hotmail.com; Alongkoad Tanomtong - tanomtong@hotmail.com; Vitaly Volobouev - vitaly@mnhn.fr; Malcolm A Ferguson-Smith - maf12@cam.ac.uk; Fengtang Yang* - fy1@sanger.ac.uk

* Corresponding author

Published: I May 2008

BMC Biology 2008, 6:18 doi:10.1186/1741-7007-6-18

This article is available from: http://www.biomedcentral.com/I74/-7007/6/18

(C) 2008 Nie et al; licensee BioMed Central Ltd.

This is an Open Access article distributed under the terms of the Creative Commons Attribution License (http://creativecommons.org/licenses/by/2.0), which permits unrestricted use, distribution, and reproduction in any medium, provided the original work is properly cited.
Received: 29 February 2008

Accepted: I May 2008

\begin{abstract}
Background: Flying lemurs or Colugos (order Dermoptera) represent an ancient mammalian lineage that contains only two extant species. Although molecular evidence strongly supports that the orders Dermoptera, Scandentia, Lagomorpha, Rodentia and Primates form a superordinal clade called Supraprimates (or Euarchontoglires), the phylogenetic placement of Dermoptera within Supraprimates remains ambiguous.

Results: To search for cytogenetic signatures that could help to clarify the evolutionary affinities within this superordinal group, we have established a genome-wide comparative map between human and the Malayan flying lemur (Galeopterus variegatus) by reciprocal chromosome painting using both human and $G$. variegatus chromosome-specific probes. The 22 human autosomal paints and the $X$ chromosome paint defined 44 homologous segments in the $G$. variegatus genome. A putative inversion on GVA II was revealed by the hybridization patterns of human chromosome probes 16 and 19. Fifteen associations of human chromosome segments (HSA) were detected in the $G$. variegatus genome: HSAI/3, I/I0, 2/2I, 3/ $21,4 / 8,4 / 18,7 / 15,7 / 16,7 / 19,10 / 16,12 / 22$ (twice), I4/I5, 16/19 (twice). Reverse painting of $G$. variegatus chromosome-specific paints onto human chromosomes confirmed the above results, and defined the origin of the homologous human chromosomal segments in these associations. In total, $G$. variegatus paints revealed 49 homologous chromosomal segments in the HSA genome.

Conclusion: Comparative analysis of our map with published maps from representative species of other placental orders, including Scandentia, Primates, Lagomorpha and Rodentia, suggests a signature rearrangement (HSA2q/2I association) that links Scandentia and Dermoptera to one sister clade. Our results thus provide new evidence for the hypothesis that Scandentia and Dermoptera have a closer phylogenetic relationship to each other than either of them has to Primates.
\end{abstract}




\section{Background}

Extensive research, based on large data sets of amino acid nuclear and mitochondrial sequences from broad mammalian taxonomic representatives, has led to a new consensus on the phylogenetic relationships of the 18 extant placental orders. Such studies have identified four superordinal clades: Afrotheria (Proboscidea, Hyracoidea, Sirenia, Tubulidentata, Macroscelidea and Arosoricida), Xenarthra (sloths, anteaters and armadillos), Supraprimates or Euarchontoglires (Rodentia, Lagomorpha, Primates, Dermoptera and Scandentia) and Laurasiatheria (Cetartiodactyla, Perissodactyla, Carnivora, Pholidota, Chiroptera and Eulipotyphla) [1-8]. However, the phylogenetic relationships among the members within each of the four superordinal clades are not well-resolved [9]. For example, a well-known case is the evolutionary affiliations of Euarchonta (= Primates + Dermoptera + Scandentia) [1], a subgroup within Supraprimates [2] (also called Euarchontoglires = Euarchonta + Glires (Rodentia + Lagomorpha)) $[1,4]$.

Within Euarchonta, the relationship between Primates, Dermoptera and Scandentia remains unresolved $[8,10-$ 12] and various hypotheses on the inter-ordinal relationships have been proposed by different morphological and molecular phylogenetic studies. In fact, all possible combinations have been proposed, including: (a) a closer relationship between Scandentia and Primates [13-15]; (b) a closer relationship between Primates and Dermoptera [1,2,8,16-19], forming the clade Primatomorpha [18]; (c) Dermoptera as a sister group to the anthropoid primates $[3,20]$; (d) Dermoptera as the closest living relative of Primates [21]; (e) a closer relationship between Scandentia and Dermoptera [4-7,22]. Furthermore, based on some intriguing similarities in morphology and molecular data, a sister-group relationship between tree shrews (Scandentia) and lagomorphs (Lagomorpha) has also been proposed by several studies $[15,23,24]$. Nevertheless, molecular and morphological studies so far have not unambiguously resolved the relationships among Primates, Dermoptera and Scandentia, suggesting a need to search for other evidence such as shared signatures provided by chromosomal rearrangements.

Cross-species chromosome painting $[25,26]$ is a powerful method for investigating the evolution of genome organizations. This method enables (1) the rapid and reliable identification of homologous chromosome segments between any two species in placental mammals based on DNA sequence homology, (2) the tracking of chromosomal rearrangements which have occurred during evolution based on the distribution pattern of conserved chromosome segments, and (3) an independent verification of the molecular phylogenetic tree using signature chromosomal rearrangements [27-34]. Chromosomal homologies between human and representative species of other placental orders have been established by cross-species chromosome painting using human chromosomespecific probes. Up to now, only two (Dermoptera and Hyracoidea) of the 18 extant placental orders have no published genome-wide comparative maps with human. While data from these orders are critical for understanding the evolution of genome organization of placental mammals as a whole, data from Dermoptera are also pivotal for the reconstruction of the ancestral karyotype and evolutionary history of all primates.

Here, for the first time, we have established the genomewide chromosomal correspondence between human and the Malayan flying lemur (Galeopterus variegatus, GVA, $2 n$ =56), one of the two living species in Dermoptera, by reciprocal cross-species chromosome painting using human and GVA probes. Comparative analysis of available comparative chromosome maps of representative species of Supraprimates has revealed cytogenetic evidence that unites the Scandentia and Dermoptera into one clade.

\section{Results \\ The G-banded karyotype and flow karyotype of $\mathbf{G}$. variegatus}

As reported previously [35], G. variegatus has a $2 n=56$ karyotype consisting of eight pairs of bi-armed (\# 2, 4-6, $8,11,13$ and 27) and 19 pairs of acrocentric autosomes (Figure 1). Chromosome 27 has a secondary constriction, the $\mathrm{X}$ chromosome is a large metacentric chromosome, and the Y chromosome is the smallest bi-armed chromosome. The G-banded karyotype of G. variegatus has not been reported previously.

The chromosomes of G. variegatus were resolved into 22 separate regions (Figure 2). Chromosome paints prepared from individual regions were hybridized to metaphases of G. variegatus, allowing the identification of chromosomes contained in each region. Except for four regions, all other regions each contained only one type of $G$. variegatus chromosomes. Of these four regions, two regions each contained two G. variegatus chromosomes (GVA8+9 and $16+17)$, the other two regions each contained three $G$. variegatus chromosomes (GVA18+19+20 and 24+25+26). A complete set of $G$. variegatus chromosome-specific paints except for the $\mathrm{Y}$ was generated.

\section{Reciprocal painting between G. variegatus and human}

To establish the genome-wide chromosomal correspondence between $G$. variegatus and human, the 22 human autosomal probes and the $\mathrm{X}$ probe were firstly hybridized onto G. variegatus chromosomes. Fluorescent in situ hybridization (FISH) examples are presented in Figure 3. The hybridization results are summarized onto a G. varie- 


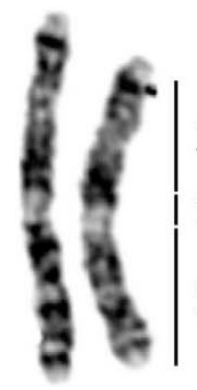

1

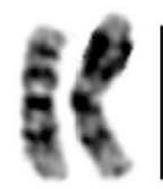

8

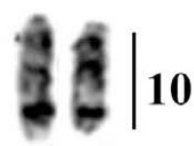

15

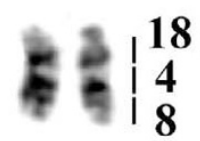

22

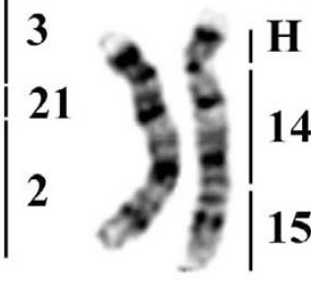

2

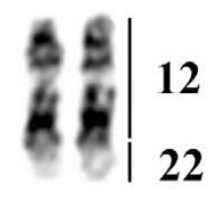

9

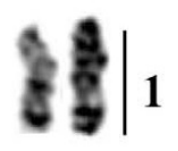

16

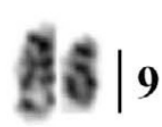

23

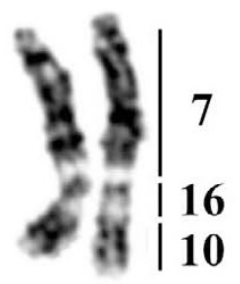

3

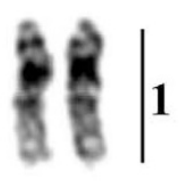

10

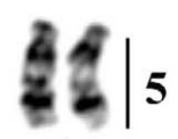

17

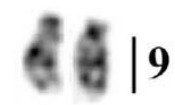

24

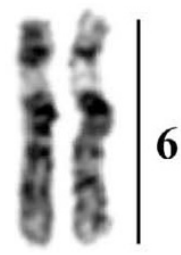

4

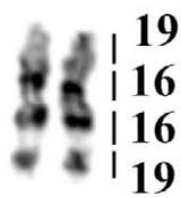

11

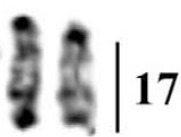

18

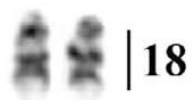

25

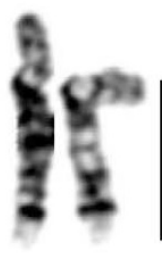

5

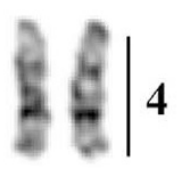

12

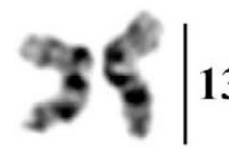

13

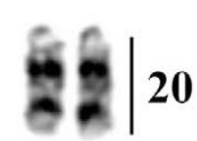

19
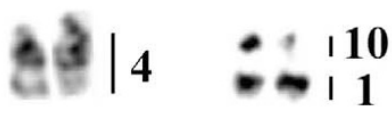

26
11
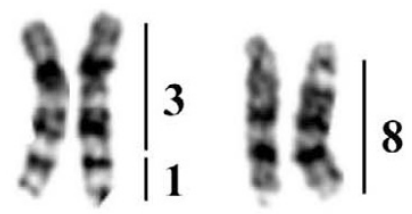

7

\section{8}

Figure I

G-banded karyotype of $G$. variegatus $(2 n=56, G V A)$, with a summary of the chromosome painting with human paints. Chromosome numbers of GVA are indicated below the chromosomes and the segments homologous to human are indicated to the right of each chromosome. H: heterochromatin.

gatus G-banded karyotype (Figure 1). Eight human chromosome probes (HSA6, 11, 13, 14, 17, 20, 21 and X) each painted one G. variegatus chromosomal segment or chromosome. Painting probes derived from 10 human chromosomes (HSA2, 3, 5, 7-9, 12, 15, 18 and 22) each hybridized onto two different G. variegatus chromosomes. Four human chromosome probes (HSA4, 10, 16, 19) each delimited three homologous G. variegatus chromosomal segments. Human chromosome probe 1 gave four signals on four G. variegatus chromosomes. The heterochromatic regions of $G$. variegatus, such as the centromeric regions of most chromosomes, the short arm of GVA2 and two parts of GVA20, were not painted by any human chromosome probe. Together, 22 human autosomal paints and the $\mathrm{X}$ chromosome paint defined 44 homologous segments in the G. variegatus genome. The hybridization patterns of human chromosome probes 16 and 19 revealed a putative inversion on GVA11. Fifteen combinations of adjacent human homologous segments, HSA1/3, 1/10, 2/21, $3 / 21,4 / 8,4 / 18,7 / 15,7 / 16,7 / 19,10 / 16,12 / 22$ (twice), $14 / 15$ and $16 / 19$ (twice), were detected in the G. variegatus genome.

To further verify these results, and to define the origin of the homologous human chromosomal segments in the segment associations detected in the G. variegatus genome, we carried out reverse painting of $G$. variegatus chromosome-specific paints onto human chromosomes. Examples are shown in Figure 3, and the results are summarized against an idiogram of the G-banded HSA karyotype (Figure 4). Eighteen G. variegatus paints (GVA4, 5, 7, $8,10,12-19,23-26$ and X) each labeled one HSA chro- 


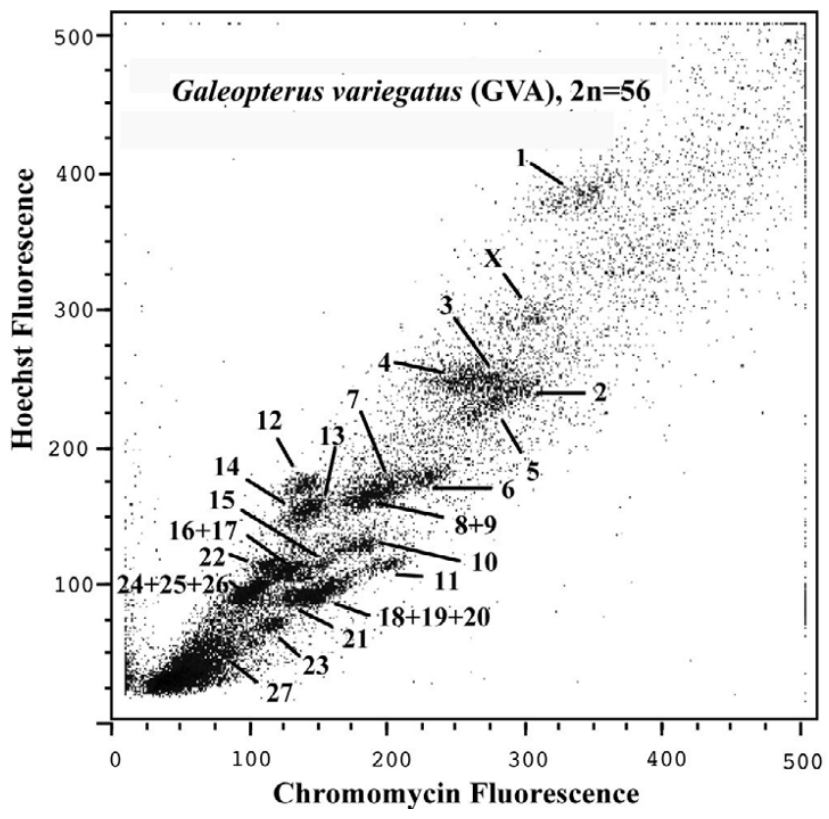

Figure 2

Bivariate flow karyotype of $\mathrm{G}$. variegatus with chromosome assignments.

mosome or chromosomal segment. Four paints (GVA2, 9, 20 and 27) each hybridized to two HSA chromosomal segments. Three paints (GVA6, 11 and 22) each painted three HSA chromosomal segments. GVA paint 1 gave hybridization signals on four HSA chromosomal segments, and GVA paints 3 and 21 each produced hybridization signals on five HSA chromosomal segments. In total, G. variegatus paints revealed 49 homologous chromosomal segments in the HSA genome.

\section{Discussion}

Resolving the phylogenetic placement of Dermoptera within Euarchonta is of fundamental importance to our understanding of the origin and evolution of human genome organization. The establishment of a genomewide comparative chromosome map between human and G. variegatus, filled our knowledge gap on the comparative genome organization of Dermoptera, and ensured that each order contained in the Euarchonta group has at least one representative species for which a genome-wide comparative map with human is available. An integrated analysis of the distribution patterns of synteny-conserved homologous segments in Dermoptera and in the representative species of Primates, Scandentia, Rodentia and Lagomorpha, enabled us to search for signature rearrangements that might provide further insight into the phylogenetic placement of Dermoptera within Euarchonta and Supraprimates.
It has been demonstrated that chromosomal rearrangements (including shared derived chromosomal syntenic associations revealed through chromosome painting) can serve as valuable phylogenetic markers to interpret the relationships of the 18 extant placental orders by the principle of parsimony $[27,29,31-34,36-40]$. When a derived chromosomal association (segments that are syntenic to two or more human chromosomes found on a single chromosome in another species) is shared by various species, this particular chromosome character (that is, a synapomorphy) may reflect a common evolutionary origin or a close phylogenetic relationship of these species $[27,28,33]$. For instance, the HSA5/21 and $1 / 19 \mathrm{p}$ in Afrotheria [31,32,39], and HSA7q/10p and 2/8 in Xenarthra [40], are considered as signature rearrangements for the superordinal clades Afrotheria and Xenarthra, respectively.

In Primates, over 70 species, including several prosimian species, have been analyzed so far by chromosome painting with human chromosome-specific probes. Except for five common ancient associations, HSA3/21, 7/16, 12/22 (twice) and 14/15, which are ancestral for all placental mammals, various landmark rearrangements have been identified for most of the nodes in primate phylogeny [41-47]. Such landmark rearrangements include HSA2/4, $4 / 6$ and $8 / 15$ associations in Lemuriform prosimians [45], HSA1q/19p, 2/12/22, 6/14, 12/7/16, 9/15 and 10/ $19 \mathrm{q}$ associations in Lorisiform prosimians [42,47], and HSA5/7, 8/18 and 10/16 in the New World monkeys [46]. In the great apes and Old World monkeys, chromosomepainting reveals that their karyotypes are highly conserved, only a few chromosomal changes differentiate their karyotypes from the ancestral karyotype of all primates [46]. However, the karyotypes of the lesser apes or gibbons dramatically differ from those of other primates in having an exceptionally high rate of chromosomal rearrangement $[43,48-52]$.

In Scandentia, the chromosomal homologies have been defined between human and a tree shrew (Tupaia belangeri, TBE, $2 n=62$ ) by reciprocal chromosome painting [53]. Results indicated that there are three derived associations (HSA2/21, 10/16 and 11/20) retained in the $T$. belangeri genome. However, a subsequent study by Richard et al revealed the existence of a HSA7 homologous segment (HSA7b), which forms part of the ancestral placental syntenic association of $H S A 7 b / 16 \mathrm{p}$, between HSA10 and 16 segments on TBE chromosome 1p [54]. Thus, the HSA10/16 association should be considered as absent in the T. belangeri genome. Instead, the association of HSA7b/10p should be considered as an additional derived character for $T$. belangeri. In Dermoptera, our chromosome painting results revealed eight additional derived human chromosomal segment associations 

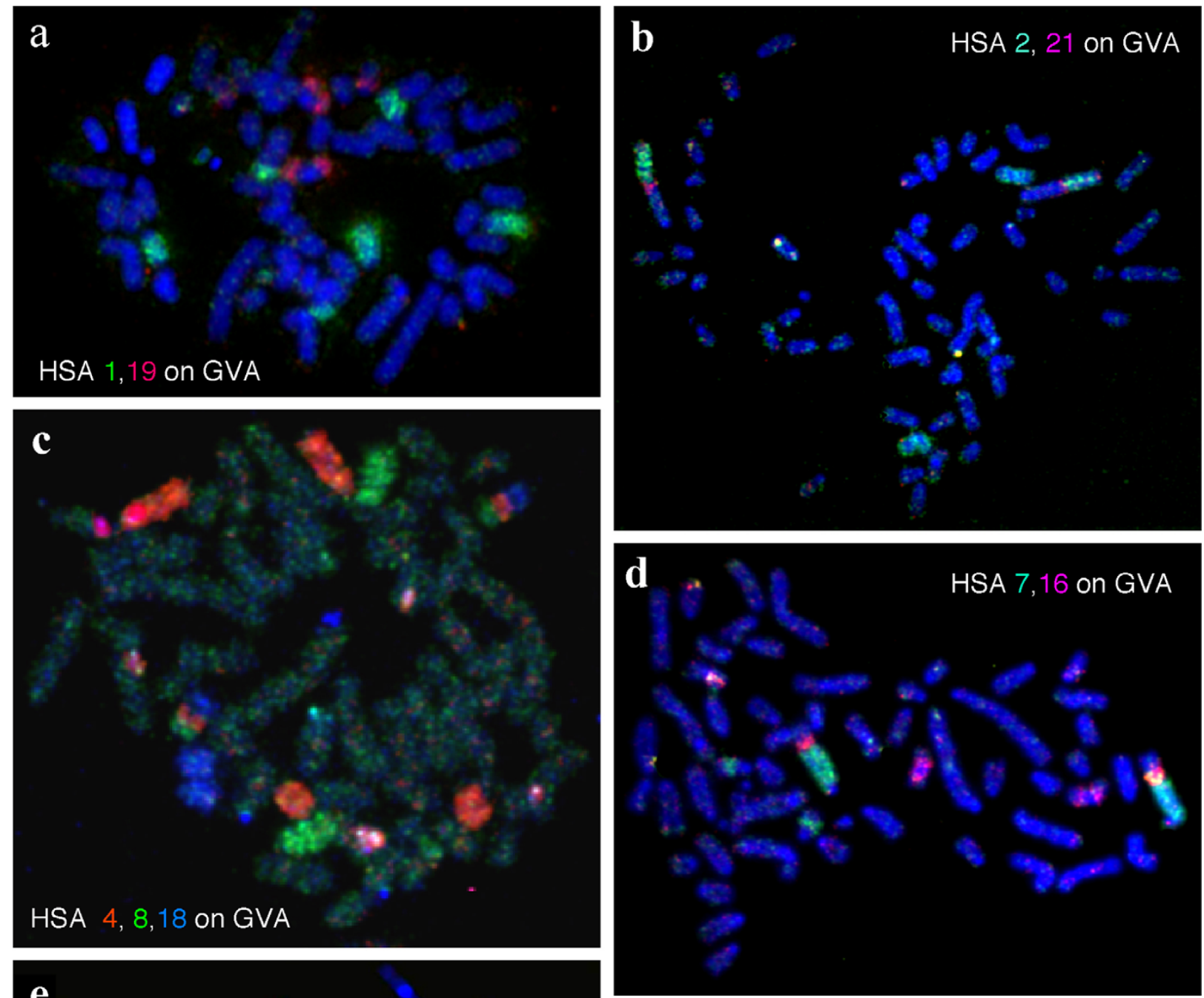

$\mathbf{e}$

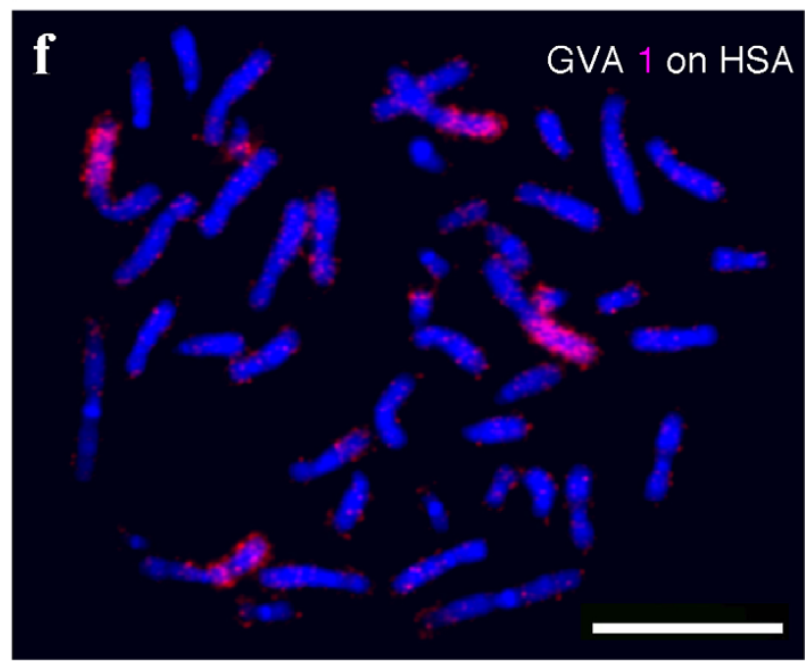

GVA 27 on HSA

Figure 3

Examples of reciprocal chromosome painting of human (HSA) probes on G. variegatus (GVA) metaphases and G. variegatus paints on human metaphases. (a)-(d) Human (HSA) probes on G. variegatus (GVA) metaphases; (e), (f) G. variegatus paints on human metaphases. 


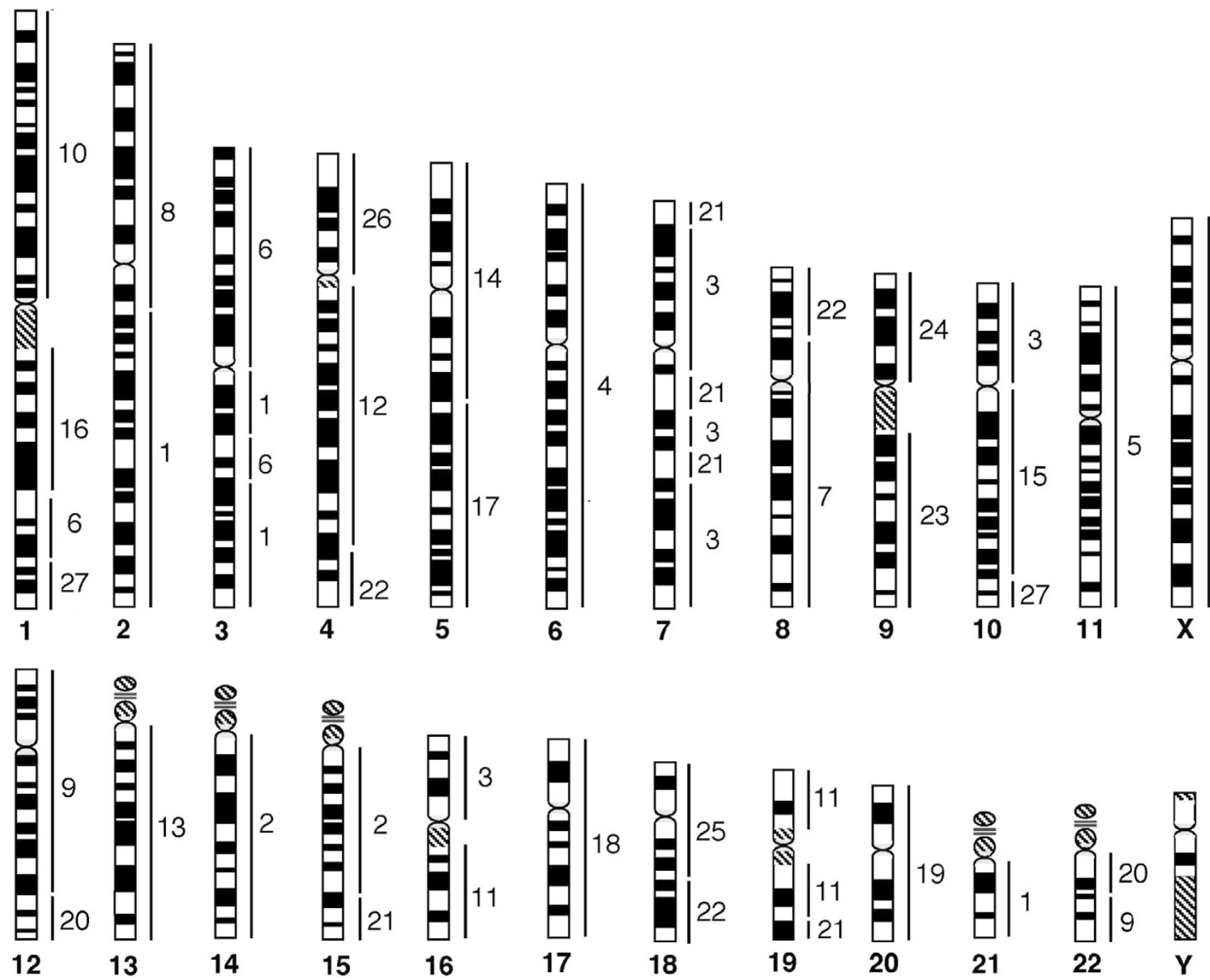

Figure 4

Summary of the chromosome painting with G. variegatus probes onto human on a G-banded idiogram. Human chromosome numbers are indicated below the chromosomes and the segments homologous to $G$. variegatus are indicated to the right of each chromosome.

(HSA1/3, 1/10, 2/21, 4/18, 7/15, 7/16p, 7/19, 10p/16p) in the genome of $G$. variegatus. Our reciprocal chromosome painting results shows that the HSA7/16 association found in $G$. variegatus is not the same as the ancestral HSA7b/16p syntenic association found in most placental mammals. The ancestral HSA7b/16p syntenic association has broken into two chromosomal segments in G. variegatus (GVA3 and 21). In Lagomorpha, the chromosome map between human and the rabbit (Oryctolagus cuniculus) has also been established by reciprocal chromosome painting [55]. In Rodentia, chromosome painting results have demonstrated that murid rodents have highly rearranged genomes $[56,57]$, while the karyotypes of squirrels are highly conserved [58-60]. Two shared derived syntenic associations between the rabbit and squirrels (HSA1/10 and 9/11) [60] have provided additional support for the clade Glires (Lagomorpha + Rodentia) [1].

We have compared the above chromosome painting data of different representative species of each order within the Supraprimates. However, we failed to detect any signature rearrangement that would support a close phylogenetic relationship either between $G$. variegatus and Primates or between Dermoptera and Glires. Although the G. variegatus and the New World monkeys share the one derived association of HSA10/16, while G. variegatus, rabbits and squirrels share the HSA1/10 association, the reverse painting results indicate that the two associations detected in $G$. variegatus and the other species had different origins. The segments contained in the HSA10/16 association of $G$. 
variegatus originated from HSA10p and 16p, while in the New World monkeys, they were derived from HSA10q and 16p [61]. In the genome of G. variegatus, the segments contained in HSA1/10 association are homologous to HSA1q and10q (Figure 4), while in the genomes of rabbits and squirrels, they are homologous to HSA1q and 10p $[55,60]$. Furthermore, comparison of the chromosome map between human and rabbits [55] with the map between human and $T$. belangeri [53] reveals no shared derived human chromosomal association that unites Scandentia and Lagomorpha into one group either.

The most salient finding comes from the comparison of the comparative chromosome map between human and $G$. variegatus with the map between human and T. belangeri. Such a comparison revealed one common derived human syntenic segment association, HSA2q/21, in both $T$. belangeri and $G$. variegatus. The results of reverse painting using the probes of $T$. belangeri and G. variegatus to hybridize human chromosomes demonstrated that HSA $2 q / 21$ association in both $T$. belangeri and G. variegatus originated from the same human homologous segments: HSA2q and 21(Figure 4 in this study, and Figure 5 in [53]). This syntenic association has not been detected in any other mammal so far studied by chromosome painting, including bats (order Chiroptera). We thus believe that the HSA2/21 association most likely represents the signature rearrangement that unites Scandentia and Dermoptera orders into one sister clade. Such a clade has been proposed previously by several molecular studies but with moderate to weak bootstrap support [4-7,22]. This grouping is in sharp contrast to a most recent molecular study that suggested the flying lemur as the closest living relative of primates [21]. It should be mentioned that so far only one representative species from each of Scandentia and Dermoptera has been studied by chromosome painting. Most species in Scandentia have a karyotype $2 n$ $=52-68$, with only one species (Urogale everetti) having $2 n$ $=44$ [62], while in Dermoptera there is the species (Cynocephalus volans) with $2 n=38$ [63]. Further chromosome painting between human and these low diploid number species will help to validate if the HSA $2 q / 21$ association is indeed the true signature rearrangement that link Scandentia and Dermoptera into one clade.

In addition to the new insight into the phylogenetic affinity of Dermoptera within Supraprimates, the establishment of the first G-banded karyotype for G. variegatus and the human-G. variegatus comparative map, together with the published human-T. belangeri comparative map, has provided us with an opportunity to compare the Gbanded karyotypes of the T. belangeri and G. variegatus in detail on the basis of genome-wide homologies defined by chromosome painting (Figure 5). Ignoring the morphological changes due to variations in the amount and distribution of heterochromatin, $12 \mathrm{G}$. variegatus autosomes (GVA1, 2, 7-9, 11, 13, 14, 17, 18, 20 and 26) show one-to-one correspondence with their $T$. belangeri counterparts; six G. variegatus autosomes (GVA10, 15, 19, 2325 ) each correspond to one $T$. belangeri autosomal segments; nine G. variegatus autosomes (GVA3-6, 12, 16, 21, 22 and 27) each correspond to between two and four $T$. belangeri chromosomes or chromosomal segments. The G-banding comparison demonstrates that most homologous chromosomes or chromosomal segments between $T$. belangeri and $G$. variegatus defined by comparative painting also show matching G-banding patterns. It is noteworthy that the acrocentric $G$. variegatus chromosome 1 (= HSA2/21/3), the largest autosome that also carries the HSA2/21 association unique for $G$. variegatus and $T$. belangeri, is conserved entirely. In contrast, G. variegatus chromosome 11 (= TBE5) that carried the ancestral placentals syntenic association of HSA16/19 has undergone an inversion as demonstrated by the chromosome painting and banding comparisons. At the current resolution level of G-banding and cross-species chromosome painting, our results suggest that simple chromosomal fissions/ fusions are the predominant mechanism that differentiates the karyotypes of $G$. variegatus and T. belangeri. However, the conservation in G-banding patterns does not necessarily indicate a close phylogenetic relationship between G. variegatus and T. belangeri. Although we have found only one interchromosomal rearrangement that unites Scandentia and Dermoptera into one clade, small and cryptic intrachromosomal rearrangements (inversions) will escape detection by the current methods. Further comparative high-resolution gene mapping in flying lemurs, tree shrews as well as outgroup species such as the rabbits, squirrels and prosimians may reveal some intrachromosomal rearrangements that could serve as additional cytogenetic evidence for the proposed Scandentia and Dermoptera sister clade.

\section{Conclusion}

We have characterized the G-banded karyotype of G. variegatus and established the first genome-wide comparative chromosome map between human and G. variegatus by reciprocal chromosome painting. Comparative analysis of the chromosome painting data from representative species of each order within the superordinal clade Supraprimates provides new molecular cytogenetic evidence that supports a sister-clade relationship between Dermoptera and Scandentia. However, our analysis has failed to identify any cytogenetic signature for well-defined clades such as the Euarchonta and Supraprimates as well as for other controversial clades. 

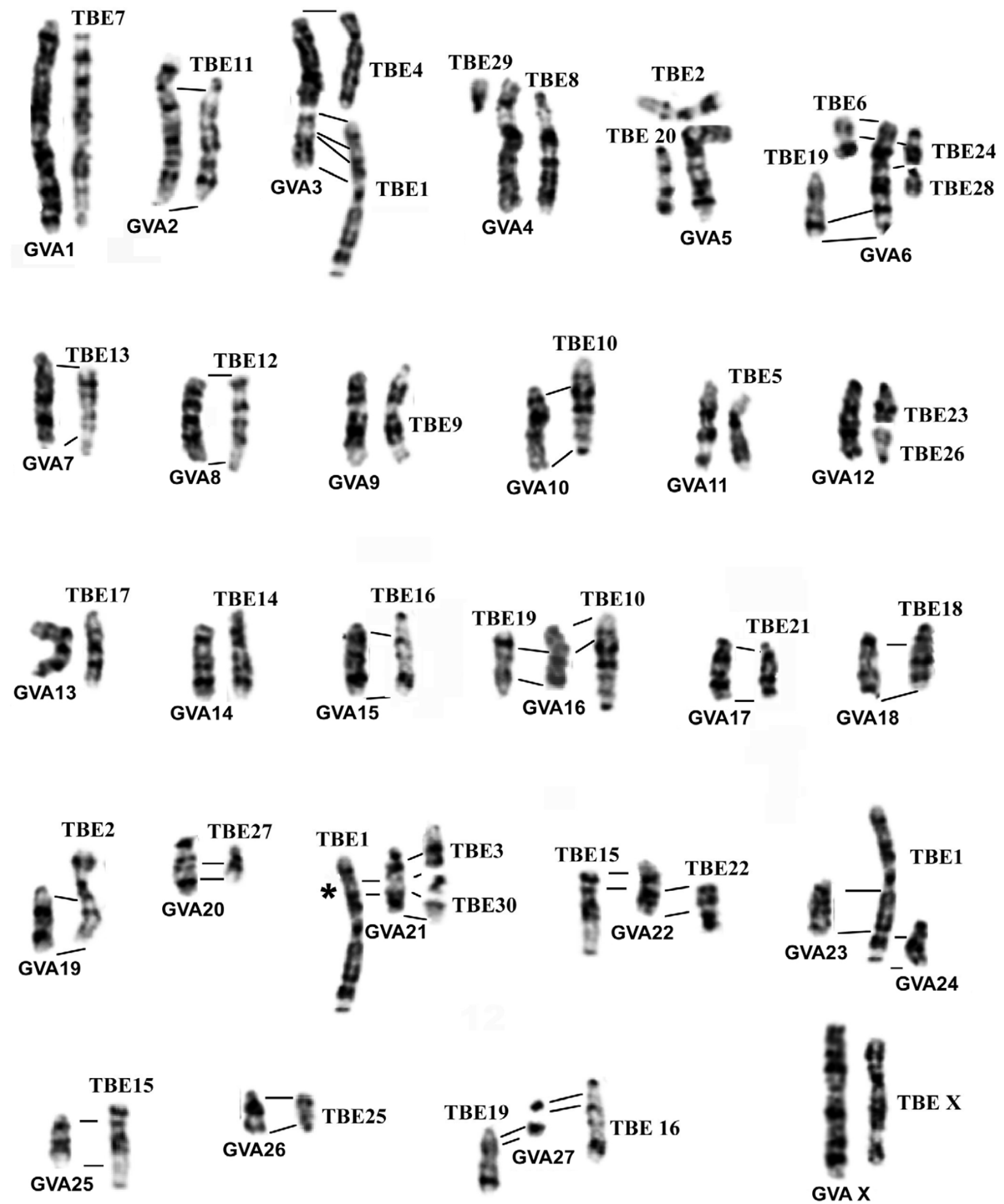

Figure 5

Correspondence between flying lemur (G. variegatus, GVA) and tree shrew (T. belangeri, TBE) G-banded chromosomes, based on chromosome painting. The flying lemur chromosome numbers are given below. The tree shrew chromosome numbers are indicated at the top, right or left. * Müller et al [53] failed to detect the human 7 homologous segment on TBE Ip, but Richard et al [30] reported its presence. 


\section{Methods}

Cell culture, metaphase preparation and G-banding

Fibroblast cell lines derived from two male G. variegatus (from Thailand) and one male tree shrew (Tupaia belangeri, TBE, $2 n=62$, KCB 200305) were established in Kunming Cell Bank, the Chinese Academy of Sciences (Kunming, Yunnan, People's Republic of China) and the Paris Natural History Museum (Paris, France) from skin biopsies. Cell culture, metaphase preparations and Gbanding were carried out following conventional methods as described previously $[31,64]$. The chromosomes of $T$. belangeri were numbered according to a previously published G-banded karyotype [53]. The karyotype of G. variegatus was arranged according to relative chromosomal size.

\section{Flow sorting and generation of chromosome-specific painting probes}

Chromosome preparation of $G$. variegatus for flow sorting followed the method described previously [26], and was sorted on a dual-laser cell sorter (FACStar Plus, Becton Dickinson) staining with chromomycin A3 $(40 \mu \mathrm{g} / \mathrm{ml}$, Sigma) and Hoechst 33258 ( $2 \mu \mathrm{g} / \mathrm{ml}$, Sigma). Chromosome-specific paints for $G$. variegatus were generated from flow-sorted chromosomes by degenerate oligonucleotide primed (DOP) polymerase chain reaction (PCR) amplification [65]. DOP-PCR-amplified chromosome-specific DNAs were labeled with biotin-16-dUTP, FITC-12-dUTP (Roche) or Cy3-dUTP (Amersham) by secondary PCR amplification. Human painting probes used in this study were also prepared from flow-sorted chromosomes as described previously [66].

\section{FISH, image capture and processing}

Reciprocal cross-species chromosome painting between human and $G$. variegatus and post-hybridization detection followed previous procedures [40,66]. Biotin-labeled probes were visualized using a layer of Cy3-avidin (1:1,000 dilution; Amersham). FITC-labeled probes were visualized using rabbit anti-FITC (1:200; DAKO) and goat anti-rabbit-FITC (1:200; Vector Laboratories). After detection, slides were mounted in Vectashield mounting medium with DAPI (4'6-diamidino-2-phenylindole, Vector Laboratories). Digital images were acquired using the CytoVision system (Applied Imaging Corp.) with a CCD camera mounted on a microscope (Olympus or Zeiss). Hybridization signals were assigned to specific chromosomes or chromosome regions defined by DAPI-banding patterns.

\section{Authors' contributions}

$\mathrm{WN}$ and BF performed chromosome-painting experiments. PCMO sorted the chromosomes. JW, WS, AT and VV collected the materials, cultured the cells, and prepared the chromosomal suspensions. WN and FY ana- lyzed the data, and wrote the paper. FY and MAFS conceived of the experiment. PCMO and MAFS revised the paper. All of the authors read and approved the final manuscript.

\section{Acknowledgements}

This study was funded in part by the National Natural Science Foundation of China (to FY and WN) and a Wellcome Trust grant (to MAFS) as well as the award of a Royal Society international short visit (to WN). The authors are grateful to Christiane Denys and Tangliang Li for helping to collect $G$. variegatus materials. FY is currently an employee of the Wellcome Trust Sanger Institute.

\section{References}

I. Waddell PJ, Okada N, Hasegawa M: Towards resolving the interordinal relationships of placental mammals. Syst Biol 1999 , 48:I-5.

2. Waddell PJ, Kishino $H$, Ota R: A phylogenetic foundation for comparative mammalian genomics. Genome Inform 200I, I 2: $14 \mid-154$.

3. Murphy WJ, Eizirik E, Johnson WE, Zhang YP, Ryder OA, O'Brien SJ: Molecular phylogenetics and the origins of placental mammals. Nature 2001, 409:614-618.

4. Murphy WJ, Eizirik E, O'Brien SJ, Madsen O, Scally M, Douady CJ, Teeling EC, Ryder OA, Stanhope MJ, de Jong WW, Springer MS: Resolution of the early placental mammal radiation using Bayesian phylogenetics. Science 200I, 294:2348-235I.

5. Liu F-GR, Miyamoto , Freire NP, Ong PQ, Tennant MR, Young TS, Gugel KF: Molecular and morphological supertrees for eutherian (placental) mammals. Science 200I, 29 I:I786-I789.

6. Springer MS, Murphy WJ, Eirizik E, O'Brien SJ: Placental mammal diversification and the Cretaceous-Tertiary boundary. Proc Natl Acad Sci USA 2003, 100:1056-1061.

7. Springer MS, Stanhope MJ, Madsen O, de Jong WW: Molecules consolidate the placental mammal tree. Trends Ecol Evol 2004, 19:430-438.

8. Bininda-Emonds ORP, Cardillo M, Jones KE, MacPhee RDE, Back RMD, Grenyer R, Price SA, Vos RA, Gittleman JL, Purvis A: The delayed rise of present-day mammals. Nature 2007, 446:507-5I 2.

9. Waddell PJ, Shelley S: Evaluating placental inter-ordinal phylogenies with novel sequences including RAGI, c-fibrinogen, ND6, and mt-tRNA, plusMCMC-driven nucleotide, amino acid, and codon models. Mol Phylogenet Evol 2003, 28:197-224.

10. Martin RD: Primate origins: plugging the gaps. Nature 1993, 363:223-234

II. Sargis EJ: New views on tree shrews: The role of Tupaiids in primate supraordinal relationships. Evol Anthropol 2004, 13:56-66.

12. Kriegs J, Churakov G, Jurka J, Brosius J, Schmitz J: Evolutionary history of 7SL RNA-derived SINEs in Supraprimates. Trends Genet 2007, 23:158-161.

13. Silcox MT, Bloch JI, Sargis EJ, Boyer DM: Euarchonta (Dermoptera, Scandentia, Primates). In The Rise of Placental Mammals Edited by: Rose KD, Archibald JD. Baltimore, MD: The Johns Hopkins University Press; 2005: 127-I44.

14. Wible JR, Covert HH: Primates: cladistic diagnosis and relationships. J Hum Evol 1987, 16: I-22.

15. Martin RD: Primate Origins and Evolution: A Phylogenetic Reconstruction London/Princeton, NJ: Chapman Hall/Princeton University Press; 1990.

16. Novacek MJ: Mammalian phylogeny: shaking the tree. Nature 1992, 356:121-125.

17. Kay RF, Thewissen JGM, Yoder AD: Cranial anatomy of Ignacius graybullianus and the affinities of the Plesiadapiformes. $\mathrm{Am} \mathrm{J}$ Phys Anthropol 1992, 89:477-498.

18. Beard KC: Phylogenetic systematics of the Primatomorpha, with special reference to Dermoptera. In Mammal Phylogeny: Placentals Edited by: Szalay FS, Novacek MJ, Mckenna MC. New York: Springer; 1993:129-150 
19. Hudelot C, Gowri-Shankar V, Jow H, Rattray M, Higgs PG: RNAbased phylogenetic methods: application to mammalian RNA sequences. Mol Phylogenet Evol 2003, 28:24I-252.

20. Arnason U, Adegoke, Bodin K, Born EW, Esa YB, Gullberg A, Nilsson M, Short RV, Xu X, Janke A: Mammalian mitogenomic relationships and the root of the eutherian tree. Proc Natl Acad Sci USA 2002, 99:8|5|-8|56.

21. Janecka JE, Miller W, Pringle TH, Wiens F, Zitzmann A, Helgen KM, Springer MS, Murphy W]: Molecular and genomic data identify the closest living relative of primates. Science 2007, 3 1 8:792-794.

22. Adkins RM, Honeycutt RL: Molecular phylogeny of the superorder Archonta. Proc Natl Acad Sci USA I99I, 88:10317-10321.

23. Bailey WJ, Slightom JL, Goodman M: Rejection of the "flying primate" hypothesis by phylogenetic evidence from the $€$ Globin Gene. Science 1992, 256:86-89.

24. Schmitz J, Ohme M, Zischler H: The complete mitochondrial genomeof Tupaia belangeri and the phylogenetic affiliation of Scandentia to other eutherian orders. Mol Biol Evol 2000, 17:1334-1343.

25. Scherthan H, Cremer T, Arnason U, Weier HU, Lima-de-Faria A, Froenicke L: Comparative chromosome painting discloses homologous segments in distantly related mammals. Nat Genet 1994, 6:342-347.

26. Yang F, Carter NP, Shi L, Ferguson-Smith MA: A comparative study of karyotypes of muntjacs by chromosome painting. Chromosoma 1995, 103:642-652.

27. Chowdhary BP, Raudsepp T, Fronicke L, Scherthan H: Emerging patterns of comparative genome organization in some mammalian species as revealed by Zoo-FISH. Genome Res 1998, 8:577-589.

28. O'Brien SJ, Menotti-Raymond M, Murphy WJ, Nash WG, Wienberg J, Stanyon R, Copeland NG, Jenkins NA, Womack JE, Marshall Graves JA: The promise of comparative genomics in mammals. Science 1999, 286:458-48।.

29. Murphy WJ, Stanyon R, O'Brien SJ: Evolution of mammalian genome organization inferred from comparative gene mapping. Genome Biol 200I, 2:reviews 0005. I-0005.8.

30. Richard F, Lombard M, Dutrillaux B: Reconstruction of the ancestral karyotype of eutherian mammals. Chromosome Res 2003, II:605-618.

31. Yang F, Alkalaeva EZ, Perelman PL, Pardini AT, Harrison WR, O'Brien PCM, Fu B, Graphodatsky AS, Robinson TJ: Reciprocal chromosome painting among human, aardvark, and elephant (superorder Afrotheria) reveals the likely eutherian ancestral karyotype. Proc Natl Acad Sci USA 2003, 100:1062-1066.

32. Froenicke L, Wienberg J, Stone G, Adams L, Stanyon R: Towards the delineation of the ancestral eutherian genome organization: Comparative genome maps of human and the African elephant (Loxodonta africana) generated by chromosome painting. Proc $R$ Soc Lond B Biol Sci 2003, 270: I33 I-1340.

33. Murphy WJ, Pevzner PA, O'Brien SJ: Mammalian phylogenomics comes of age. Trends Genet 2004, 20:631-639.

34. Froenicke L: Origins of primate chromosomes - as delineated by Zoo-FISH and alignments of human and mouse draft genome sequences. Cytogenet Genome Res 2005, 108:122-138.

35. Hsu TC, Benirschke K: An Atlas of Mammalian Chromosomes Volume 7. Berlin: Springer; 1973:339-340.

36. Ferguson-Smith MA, Yang F, O'Brien PCM: Comparative mapping using chromosome sorting and painting. ILARJ 1998, 39:68-76.

37. Wienberg J, Stanyon R: Comparative chromosome painting of primate genomes. ILAR J I998, 39:77-9I.

38. Yang F, O'Brien PC, Milne BS, Graphodatsky AS, Solanky N, Trifonov $V$, Rens W, Sargan D, Ferguson-Smith MA: A complete comparative chromosome map for the dog, red fox and human and its integration with canine genetic maps. Genomics 1999, 62:189-202.

39. Robinson TJ, Fu B, Ferguson-Smith MA, Yang F: Cross-species chromosome painting in the golden mole and elephantshrew: support for the mammalian clades Afrotheria and Afroinsectiphillia but not Afroinsectivora. Proc R Soc Lond B Biol Sci 2004, 27 I: 1477-84.

40. Yang F, Graphodatsky AS, Li T, Fu B, Dobigny G, Wang J, Perelman PL, Serdukova NA, Su W, O'Brien PCM, Wang Y, Ferguson-Smith MA, Volobouev V, Nie W: Comparative genome maps of the pangolin, hedgehog, sloth, anteater and human revealed by cross-species chromosome painting: further insight into the ancestral karyotype and genome evolution of eutherian mammals. Chromosome Res 2006, 14:283-296.

4I. Müller S, O'Brien PCM, Ferguson-Smith MA, Wienberg J: Reciprocal chromosome painting between human and prosimians (Eulemur macaco macaco and E. fulvus mayottensis). Cytogenet Cell Genet 1997, 78:260-27I.

42. Stanyon R, Koehler U, Consigliere S: Chromosome painting reveals that galagos have highly derived karyotypes. Am J Phys Anthropol 2002, I I 7:319-326.

43. Müller S, Hollatz M, Wienberg J: Chromosomal phylogeny and evolution of gibbons (Hylobatidae). Hum Genet 2003, I I 3:493-50|.

44. Ferguson-Smith MA, Yang F, Rens W, O'Brien PCM: The impact of chromosome sorting and painting on the comparative analysis of primate genomes. Cytogenet Genome Res 2005, 108:|| 2-2|.

45. Warter S, Hauwy M, Dutrillaux B, Rumpler Y: Application of molecular cytogenetics for chromosomal evolution of the Lemuriformes (Prosimians). Cytogenet Genome Res 2005, 108:197-203

46. Wienberg J: Fluorescence in situ hybridization to chromosomes as a tool to understand human and primate genome evolution. Cytogenet Genome Res 2005, 108:139-160.

47. Nie W, Fu B, O'Brien PCM, Wang W, Su W, Robinson TJ, Yang F: Chromosome painting between human and lorisiform prosimians: evidence for the HSA 7/16 synteny in the primate ancestral karyotype. Am J Phys Anthropol 2005, 129:250-259.

48. Jauch A, Weinberg J, Stanyon R, Arnold N, Tofanelli S, Ishida T, Cremer $\mathrm{T}$ : Reconstruction of genomic rearrangements in great apes and gibbons by chromosome painting. Proc Natl Acad Sci USA 1992, 89:861I-86I5.

49. Koehler U, Arnold N, Wienberg J, Tofanelli S, Stanyon R: Genomic reorganization and disrupted chromosomal synteny in the siamang (Hylobates syndactylus) revealed by fluorescence in situ hybridization. Am J Phys Anthropol 1995, 97:37-47.

50. Koehler U, Bigoni F, Wienberg J, Stanyon R: Genomic reorganization in the concolor gibbon (Hylobates concolor) revealed by chromosome painting. Genomics 1995, 30:287-292.

5I. Yu D, Yang F, Liu R: A comparative chromosome map between human and Hylobates hoolock built by chromosome painting. Acta Genetica Sinica 1997, 24:417-23.

52. Nie W, Rens W, Wang J, Yang F: Conserved chromosome segments in Hylobates hoolock revealed by human and $H$. leucogenys paint probes. Cytogenet Cell Genet 200I, 92:248-253.

53. Müller S, Stanyon R, O'Brien PCM, Ferguson-Smith MA, Plesker R, Wienberg J: Defining the ancestral karyotype of all primates by multidirectional chromosome painting between tree shrews, lemurs and humans. Chromosoma 1999, 108:393-400.

54. Richard F, Lombard M, Dutrillaux B: Phylogenetic origin of human chromosomes 7,16 , and 19 and their homologs in placental mammals. Genome Res 2000, 10:644-65I.

55. Korstanje R, O'Brien PC, Yang F, Rens W, Bosma AA, van Lith HA, van Zutphen LF, Ferguson-Smith MA: Complete homology maps of the rabbit (Oryctolagus cuniculus) and human by reciprocal chromosome painting. Cytogenet Cell Genet 1999, 86:317-322.

56. Stanyon R, Yang F, Cavagna P, O'Brien PC, Bagga M, Ferguson-Smith MA, Wienberg J: Reciprocal chromosome painting shows that genomic rearrangement between rat and mouse proceeds ten times faster than between humans and cats. Cytogenet Cell Genet 1999, 84:150-I55.

57. Stanyon R, Yang F, Morescalchi AM, Galleni L: Chromosome painting in the long-tailed field mouse provides insights into the ancestral murid karyotype. Cytogenet Genome Res 2004, 105:406-4II.

58. Richard F, Messaoudi C, Bonnet-Garnier A, Lombard M, Dutrillaux B Highly conserved chromosomes in an Asian squirrel (Menetes berdmorei, Rodentia:Sciuridae) as demonstrated by ZOO-FISH with human probes. Chromosome Res 2003, I I:597-603

59. Stanyon R, Stone G, Garcia M, Froenicke L: Reciprocal chromosome painting shows that squirrels, unlike murid rodents, have a highly conserved genome organization. Genomics 2003, 82:245-249. 
60. Li T, O'Brien PC, Biltueva L, Fu B, Wang J, Nie W, Ferguson-Smith MA, Graphodatsky AS, Yang F: Evolution of genome organizations of squirrels (Sciuridae) revealed by cross-species chromosome painting. Chromosome Res 2004, I 2:317-335.

61. Stanyon R, Consigliere S, Bigoni F, Ferguson-Smith, O'Brien PCM, Wienberg J: Reciprocal chromosome painting between a New World primate, the woolly monkey, and humans. Chromosome Res 2001, 9:97-106.

62. Chu EHY, Bender MA: Cytogenetics and evolution of primates. Ann N Y Acad Sci 1962, 102:253-266.

63. Richart EA: Chromosomes of Philippine mammals (Insectivora, Dermoptera, Primates, Rodentia, Carnivora). Proc Biol Soc Wash 2003, I I 6:699-709.

64. Nie W, Wang J, O'Brien PCM, Fu B, Tian Y, Ferguson-Smith MA, Yang $F$ : The genome phylogeny of domestic cat, red panda and five mustelid species revealed by comparative chromosome painting and G-banding. Chromosome Res 2002, 10:209-222.

65. Telenius H, Pelmear AH, Tunnacliffe A, Carter NP, Behmel A, Ferguson-Smith MA, Nordenskjold M, Pfragner R, Ponder BA: Cytogenetic analysis by chromosome painting using DOP-PCR amplified flow-sorted chromosomes. Genes Chromosomes Cancer 1992, 4:2576-2263.

66. Yang F, Müller S, Just R, Ferguson-Smith MA, Wienberg J: Comparative chromosome painting in mammals: human and the Indian muntjac (Muntiacus muntjak vaginalis). Genomics 1997, 39:396-401.

Publish with Bio Med Central and every scientist can read your work free of charge

"BioMed Central will be the most significant development for disseminating the results of biomedical research in our lifetime. "

Sir Paul Nurse, Cancer Research UK

Your research papers will be:

- available free of charge to the entire biomedical community

- peer reviewed and published immediately upon acceptance

- cited in PubMed and archived on PubMed Central

- yours - you keep the copyright

Submit your manuscript here:

http://www.biomedcentral.com/info/publishing_adv.asp
BiolMedcentral 